\title{
Cupper doping effect on the electrical characteristics of TiO2 based Memristor
}

\author{
Banafsheh Alizadeh Arashloo ${ }^{\mathrm{a}}$, Hasan Efeoglu ${ }^{\mathrm{a}, \mathrm{b}}$ \\ ${ }^{a}$ Department of Nanoscience and Nanoengineering, Ataturk University, 25030Erzurum/Turkey \\ ${ }^{b}$ Ataturk University, Faculty of Engineering, Department of Electric \& Electronics Eng. 25030 Erzurum TURKEY
}

\section{Keywords}

\section{Memristor,}

Cu doped,

$\mathrm{TiO}_{2}$,

Hysteresis loop.

\begin{abstract}
Nanostructures as a starting point to solve the scaling problems of the CMOS technologies, have been concerned the attention of numerous researchers. By strong demanding for nonvolatile memory technology, resistive memories based on metal oxide has been common due to several advantages, such as low-power consumption, good scalability and fast switching speed. Even though high-temperature fabrication process has a large area limitation by their material characteristics. Metal oxide thin films are respectable candidate to fabricate at nano scale solid state electronic device. Metal/Metal-Oxide/Metal structure is employed to several devices such as Non-volatile able memories, RRAMs, resistance switching based devices and memristor. The foundation of the primary TiO2 based memristor served a number of consequences for understanding the conduction mechanisms during the formation of hysteresis loop. Also, the current-voltage characteristics (hysteretic loop) which is formed by mobile anions or oxygen vacancies motion in the set and reset process, is clarified the resistive switching behavior by swapping the resistance of TiO2 thin film. Here, the effect of $\mathrm{Cu}$ doping into TiO2 based memristor by focused on the hysteresis loop characteristics is considered. Similarities of hysteresis loop form in $\mathrm{Cu}$ doped devices are explored. Hysteresis loop is symmetric for structures having pure TiO2; however, asymmetric character appears after $\mathrm{Cu}$ doping. After the formation process hysteresis loop of the $\mathrm{Cu}$ doped devices shown higher conductance path on (I-V) characteristic than the initial forming process loop in positive cycle loop as the un-doped TiO2. Also, in spite of undoped $\mathrm{TiO} 2$, this (I-V) hysteresis loop character shown lower path conductance than primary forming process in the negative cycle loop. Surface roughness of $30 \mathrm{~nm}$ thick TiO2 is increased from $0.3 \mathrm{~nm}$ to $0.77 \mathrm{~nm}$ as $\mathrm{Cu}$ doping increased from $\% 10$ to $\% 30$. Unfortunately, $\mathrm{XRD}$ results cleared that there is no exchange in crystallinity but optical band gap decreased as $\mathrm{Cu}$ doping increased.
\end{abstract}

\section{Introduction}

Initially memristor theory is proposed by Leon Chua [1]. Up today, its electrical characteristics have been the focus point of researchers. Memristor as a forth elements of electronics have resistance switching properties which is promising next generation nonvolatile memory due to its artless structure, simple processing, fast switching and high-density fabrication. Charge-based memory devices such as flash memory and dynamic random-access memory (DRAM), have physical and technological confinement when the device sizes have minimized. Therefore, switching mechanism centered on resistance change is an alternative solution to overcome device dimensions shrunk [2-5]. Several semiconductor materials and oxides or insulators have been discovered to reveal resistive switching properties, such as chalcogenides [6,7], organic materials [8,9], amorphous silicon [10,11], perovskite oxides [12,13], binary transition metal oxides (such as $\mathrm{NiO}$ [14-16], $\mathrm{TiO}_{2}$ [17-19], $\mathrm{ZnO}$ [19]) and graphene oxide (GO) thin films [20]. Among the various of candidate materials to select, two terminal metal oxide based resistive switching memories due to advantages includes, low-power consumption, scalability down to nano scale, and fast switching speed are promising for future electronics. $\mathrm{Pt} / \mathrm{TiO}_{2} / \mathrm{Pt}$ as the first structure of the memristor introduced by HP [21]. Recently, a developing computing paradigm, neuromorphic computing which is based on working principles of neural systems in the brain, attracted the most special attention about memristor structures. In the other hand, learning activities in the brain memory are processed by dynamic mass changes and spikes occurrences between the neural networks. This progression is titled as synaptic plasticity. However, it is not simple which common memristors gradually executed the mimic of the bio synapse function and conduction but the doped oxide based memristor as have reported in literature can indeed emulate [23-26]. As an example, selfassembled $\mathrm{Ag}$ nanoclusters in $\mathrm{TiO}_{2}$ films are employed $\mathrm{Ag}$ dopant gradient to boost the memristor enhancement. Under the negative and positive pulse trains, memristor is revealed respectively high and low resistance switching, which can completely match with stimulus and blockage spike of the bio synapse [27]. Also, self-doping memristors introduced to emulate the ion dynamics $\left(\mathrm{Ca}^{2+}\right)$ of biological synapses in $\mathrm{Ag} / \mathrm{Ag}: \mathrm{Ta}_{2} \mathrm{O}_{5} / \mathrm{Pt}$ structure [28]. Another work is a penetrate $\mathrm{Ag}$ into oxide of $\mathrm{Pt} / \mathrm{SiO}_{\mathrm{x}} \mathrm{N}_{\mathrm{y}}: \mathrm{Ag} / \mathrm{Pt}$ device. In this study $\mathrm{Ag}$ filament formation/captured could emulate $\mathrm{Ca}^{2+}$ mimic of bio synapses. There are many studies about synapse functions emulation by emerging devices such as memristor-based synapses, phase change synapses, ferroelectric synapses, 2D material synapses, and metal-oxidesemiconductor (CMOS) based artificial synapses. In neuromorphic engineering, CMOS based artificial synapses circuits have high power consumption due to multipart structure needs. The most attractive candidate among these structures are two terminal memristor which is exactly identical synapses [28]. Low power consumption and also three-dimensional scalability of memristors have advantages over conventional CMOS technology [30-31]. Consequently, the investigation of doping effect on the electrical characteristics of memristors is important agenda which is noticeable for bios naps emulation modeling. Here, it is focused on the $\mathrm{Cu}$ doped $\mathrm{TiO}_{2}$ based memristor. The current-voltage characteristic of $\left(\mathrm{Al} / \mathrm{p}^{++} \mathrm{Si} / \mathrm{Cu}+\mathrm{TiO}_{2}\right.$ or 
$\mathrm{TiO}_{2} / \mathrm{Cu}$ ) structure devices are considered by adding $\mathrm{Cu}$. $\mathrm{Cu}$ doping effect on electrical parameters of these memristors are investigated.

\section{Experimental Setup and Measurements}

The $\mathrm{Al} / \mathrm{p}^{++} \mathrm{Si} /\left(\mathrm{TiO}_{2}\right)$ or $\left(\mathrm{TiO}_{2}+\mathrm{Cu}\right) / \mathrm{Cu}$ structures (as shown in Fig1.a, b. processed on a $\mathrm{p}^{++} \mathrm{Si}$ wafer as a memristor by HiPIMS technique. At the first step inorganic and organic cleaning process called as RCA1 and RCA2 applied to silicon wafers. Thin $\mathrm{SiO}_{2}$ film is grown at $1000^{\circ} \mathrm{C}$ for after thermal evaporation $\mathrm{Al}(1200 \AA)$ and annealing at $5800^{\circ} \mathrm{C} / 5 \mathrm{~min}$, under the Argon atmosphere. Lithography process through oxide layer is used for windows opening. Lift-Off process used for $\mathrm{TiO}_{2}$ or $\mathrm{TiO}_{2}+\mathrm{Cu}$ growth and top metal deposition, Fig1.a, b. Device size was $100 \times 100 \mu \mathrm{m}^{2}$. Output of homemade pulsed DC source was set to $580 \mathrm{~V}$ and 650V respectively for $\mathrm{Ti}$ and $\mathrm{Cu}$ targets, with pulse durations equal to 50ms. On the other hand, the pulse frequency of applied voltage to $\mathrm{Ti}$ target was $5 \mathrm{~Hz}$. According to thickness measurements of $\mathrm{TiO}_{2}(500 \AA)$ and $\mathrm{Cu}$ grown rate ratio on time, the DC pulse frequency which is applied to $\mathrm{Cu}$ target in the sputtering chamber choose as $290,435,580,725,870 \mathrm{mHz}$, respectively for $10,15,20,25,30 \% \mathrm{Cu}$ doping as shown in the Fig1. c. Ar and $\mathrm{O}_{2}$ flow rates set to $30 \mathrm{sccm}$ and $0.4 \mathrm{sccm}$ respectively. Initial base pressure was below $5 \times 10^{-5}$ Torr. During the deposition the base pressure was $5.13 \times 10^{-3}$ Torr. Fiber optic coupled Ocean HR4000 spectrometer used for plasma emission monitoring during the film growth and later used for transmission measurements. Time depended (I-V-t) measurements were carried out by a computer controlled Keithley 2400 and 6514 at room temperature. Films grown on $\mathrm{SiO}_{2} / \mathrm{p}^{++} \mathrm{Si}$ were analyzed using Rigaku DMax-2200XRD with a $\mathrm{Cu}-\mathrm{K} \alpha(\lambda: 1.5405 \AA)$ radiation source (at $20-60$ scan range). AFM measurements for surface morfology were carried out by using AFM NanoMagnetics Instruments.

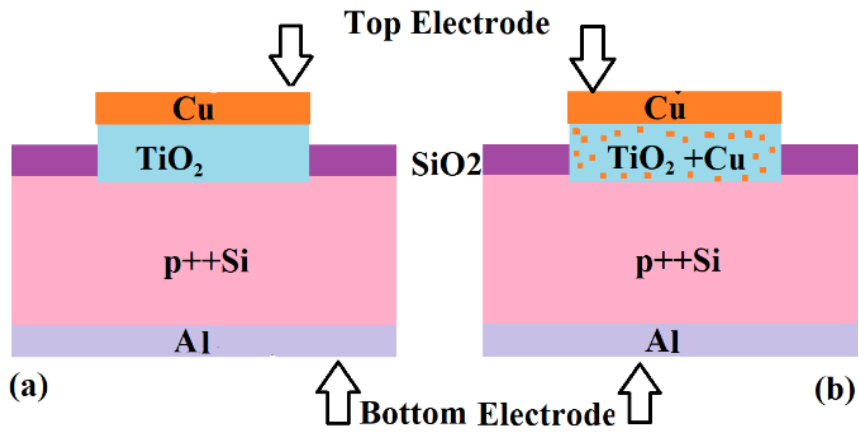

applied frequency to $\mathrm{Ti} \& \mathrm{Cu}$
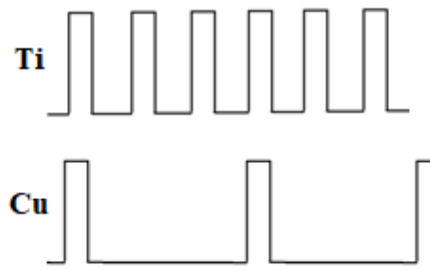

$\mathbf{f}=\mathbf{5 H z}$

$\mathrm{f}=290,435,580,725,870 \mathrm{mHz}$ $10 \mathrm{~min}$ and under the dry oxygen flow. Back ohmic contact is formed

the oxygen ions or vacancy. Memristor resistance is decreased after each loop, Fig.2a, b, c. Performed R-t measurement is carried out at $+2 \mathrm{~V}$ and $-1.5 \mathrm{~V}$. Additionally, symmetric I-V) character recognized in the un doped $\mathrm{TiO}_{2}$ device.
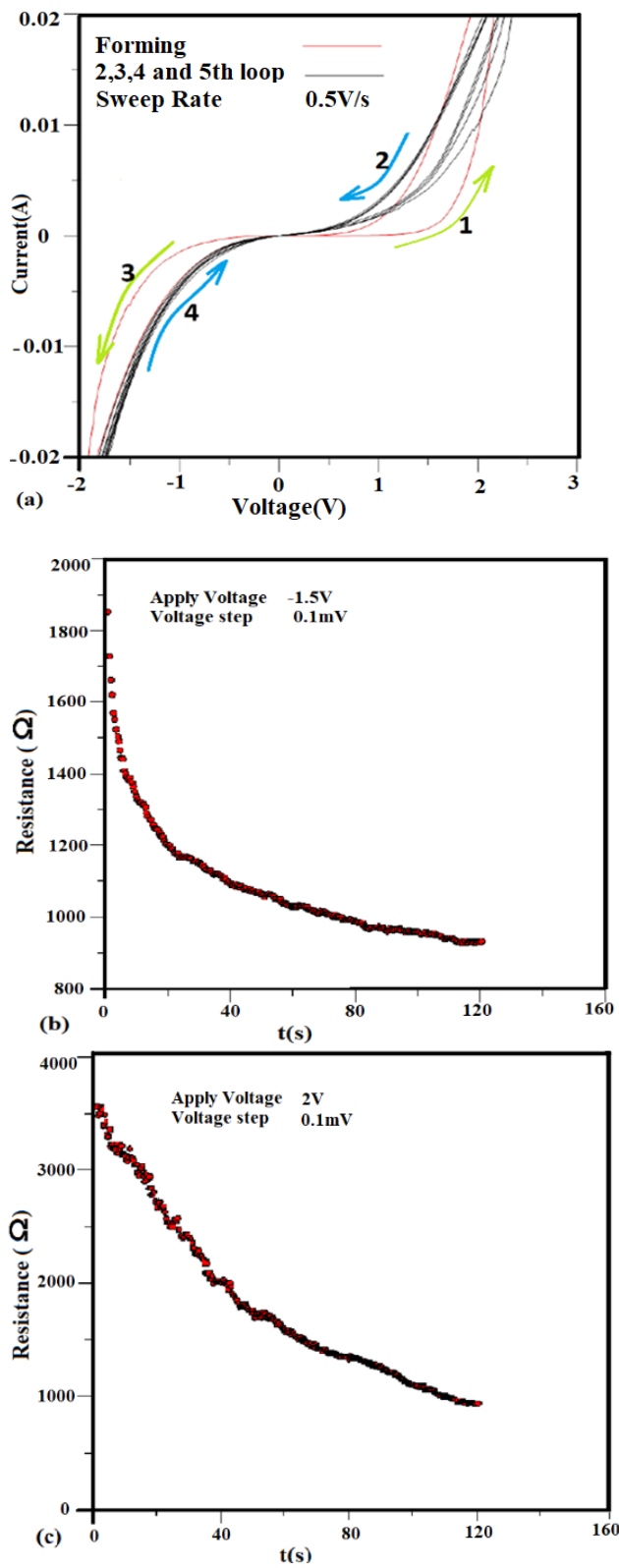

Figure 1. Schematics of (a) $\left(\mathrm{Al} / \mathrm{p}^{++} \mathrm{Si} / \mathrm{TiO}_{2} / \mathrm{Cu}\right)$ device, (b) $\left(\mathrm{Al} / \mathrm{p}^{++} \mathrm{Si} /\left(\mathrm{TiO}_{2}+\% \mathrm{kCu}\right) / \mathrm{Cu}\right)$ device, $10 \%<\mathrm{k}<30 \%$ (c)DC pulse timing applied to $\mathrm{Ti}$ and $\mathrm{Cu}$ targets from HiPIMS power supplies.

\section{a. Memristor characteristics (I-V and R-t)}

As grown structures of $\mathrm{Al}(1200 \AA) / \mathrm{p}^{++} \mathrm{Si} / \mathrm{TiO}_{2}(500 \AA)$ and $\left(\mathrm{TiO}_{2}+\mathrm{Cu}\right) / \mathrm{Cu}(500 \AA)$ devices is shown in Fig.1a. During every I-V-t measurement sweep rate was equal to $0.5 \mathrm{~V} / \mathrm{sec}$, and compliance current was $20 \mathrm{~mA}$ during each cycle. Forming process is seen in Fig.2a. The set voltage of forming was approximately $1.5 \mathrm{~V}$ which increased by repetition loop number. According to measurement results in Fig.2a, after each cycle, the hysteresis loop area is shrunk. In Fig.2a the set/reset of memristor which is known as resistive switching, this mechanism is defined and to be explained by drift of
Figure 2. (I-V) characteristics of the $\left(\mathrm{Al} / \mathrm{p}^{++} \mathrm{Si} / \mathrm{TiO}_{2} / \mathrm{Cu}\right)$ device. (a)The forming process in the sweep rate $0.5 \mathrm{~V} / \mathrm{s}$. Resistance change under the constant voltage applied as (b) $+2 \mathrm{~V}$ and (c) $-1.5 \mathrm{~V}$

As shown in the Fig.3, the set voltage pointed in the pure and $\mathrm{Cu}$ doped $\mathrm{TiO}_{2}$ is performed individually and then plotted together. The comparison of set voltages appears during the forming process of doped and un-doped $\mathrm{TiO}_{2}$ memristor is shown in Fig.3. Addition of $\mathrm{Cu}$ into $\mathrm{TiO}_{2}$ layer the set point is formed at higher voltages. The increase set voltages are completed approximately as a sharp filament formation while loop progressing. Although repeated measurements do not show efficient stability in switching process, but the forming process is acknowledged by accumulative of $\mathrm{Cu}$ which is doped to $\mathrm{TiO}_{2}$ during the film growth. 


\subsection{Cu Doped Memristor's Characteristics (I-V and R-t)}

The (I-V) characteristics of pure and 10,15,25\% Cu doped $\mathrm{TiO}_{2}$ based memristor is shown in Fig. 4a,b,c,d where sweep rate was $0.1 \mathrm{~V} / \mathrm{s}$ and compliance current was $20 \mathrm{~mA}$. In spite of the pure $\mathrm{TiO}_{2}$ based device an asymmetric (I-V) is achieved after $\mathrm{Cu}$ doping into $\mathrm{TiO}_{2}$, Fig.4. Repeated loops are clarified improvement of resistance in the negative cycle and reducing the resistance in positive cycle of hysteresis. In the other words, by $\mathrm{Cu}$ impurity increase, the device is converted to an inverter. To support the recurrence performance of resistance switching behavior of memristor, resistance measurement is done. As shown in the Fig. $5 \mathrm{a}$, $\mathrm{b}$ resistance increase under the $+2 \mathrm{~V}$ bias and resistance decrease under the $-4 \mathrm{~V}$ supports the observation given in Fig. 4c.
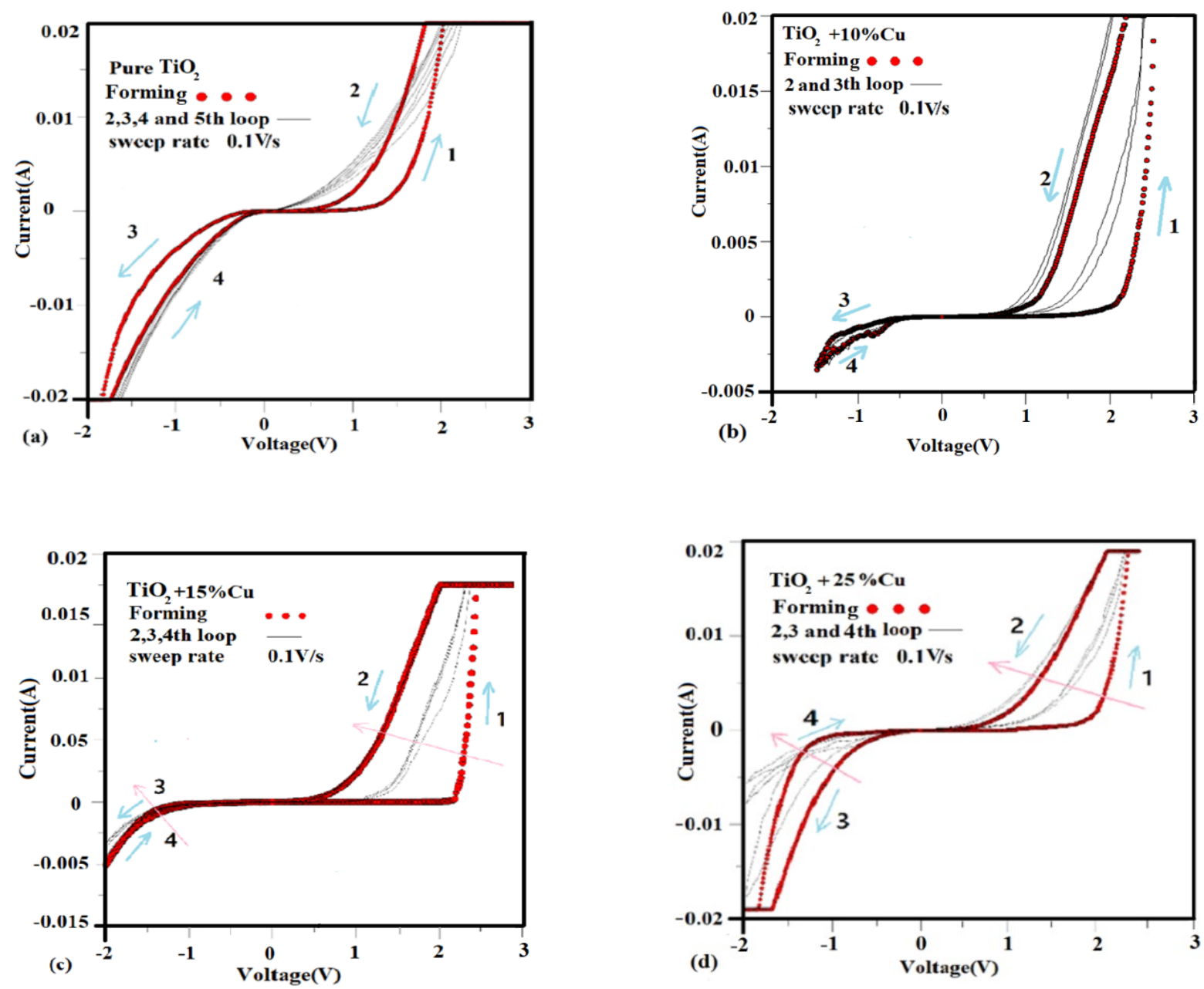

Figure 4. (I-V) characteristics of memristor of (a)un doped $\mathrm{TiO}_{2}$, (b) $10 \% \mathrm{Cu}$ doped device, (c) $15 \% \mathrm{Cu}$ doped device and (d) $25 \% \mathrm{Cu}$ doped device. The result shown the asymmetric I-V relation by addition $\mathrm{Cu}$ impurity. Resistance increase during the positive cycle and decrease during the negative cycle property clearly appear after \%25 Cu doping. 


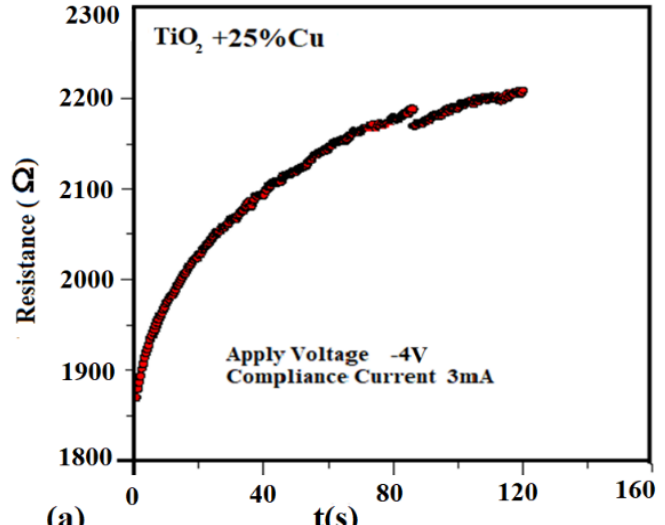

(a)

$\mathbf{t}(\mathbf{s})$
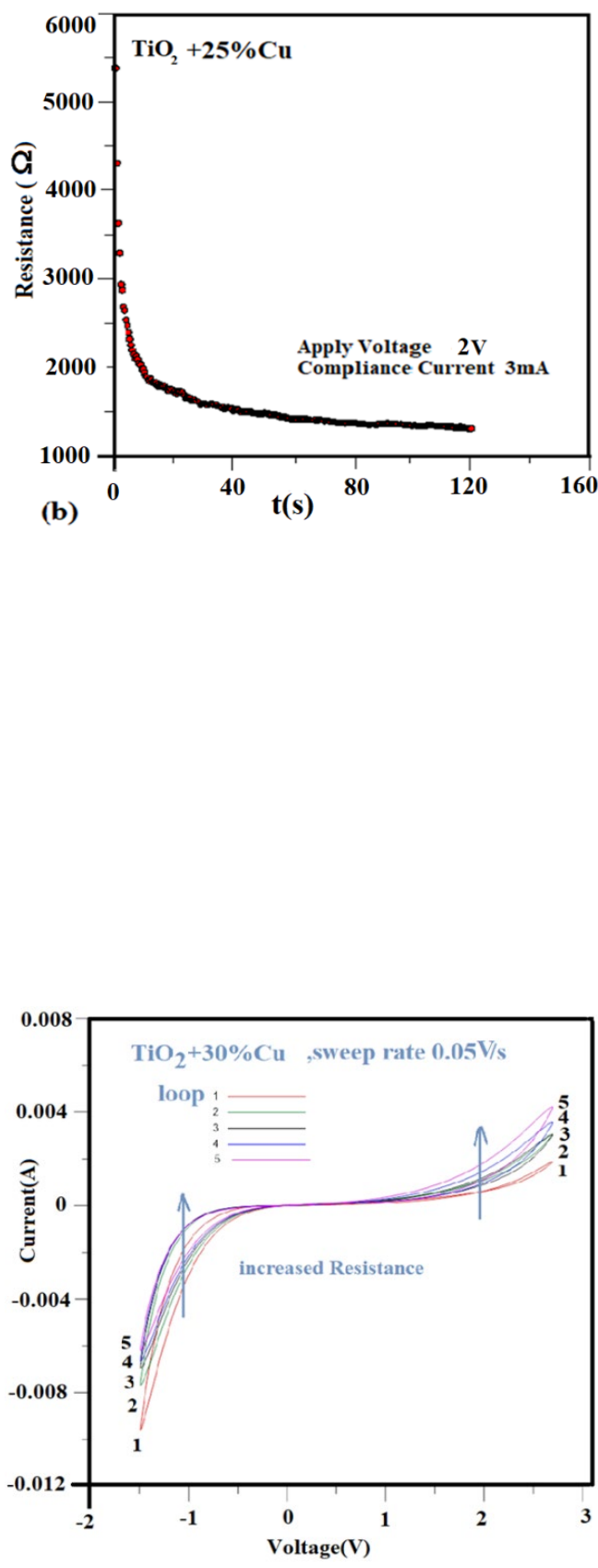

Figure 6. (I-V-t) characteristics of $30 \% \mathrm{Cu}$ doped memristor. Current increase during the positive cycle and current decrease during the negative cycle after each loop is characteristic property of memristor.
The XRD results of $\mathrm{TiO}_{2}$ and $\mathrm{Cu}$ doped $\mathrm{TiO}_{2}$ layers on silicon substrate by R-HiPIMS, are shown in Fig.6. As shown in this figure, the peak labelled as " $T$ " in XRD plots are related to titanium dioxide or $\mathrm{Ti}_{8} \mathrm{O}_{15}$ at $2 \theta=33.21^{\circ}$, and the other peaks are related to brookite $\mathrm{TiO}_{2}(\mathrm{~B}),(\mathrm{A}, \mathrm{R})$ $\mathrm{Ti}_{8} \mathrm{O}_{15}$ rutile and anatase phases. Peak donated with "C" to be related to compound of Titanium, $\mathrm{O}$ and $\mathrm{Cu}\left(\mathrm{Cu}_{3} \mathrm{Ti}_{4} \mathrm{O}\right.$ and $\left.\mathrm{Cu}_{3} \mathrm{TiO}_{4}\right)$. As reported, $\mathrm{Cu}$-doped(1.7-3.2\%Cu) $\mathrm{TiO}_{2}$ nanorods, have ferromagnetic properties. The XPS analyses shows that ferromagnetic property is centered on existence of oxygen vacancies which improvement by $\mathrm{Cu}$ doping. In same study, as reported, highly crystalline anatase phase is clarified in the $\mathrm{Cu}$ doped nanorods by high-resolution transmission electron microscopy (HRTEM) analyses [32]. Therefore, XRD results given in Fig. 6 is supported by literature which, $\mathrm{Cu}$ doping has negligible effect on crystallinity of $\mathrm{TiO}_{2}$.

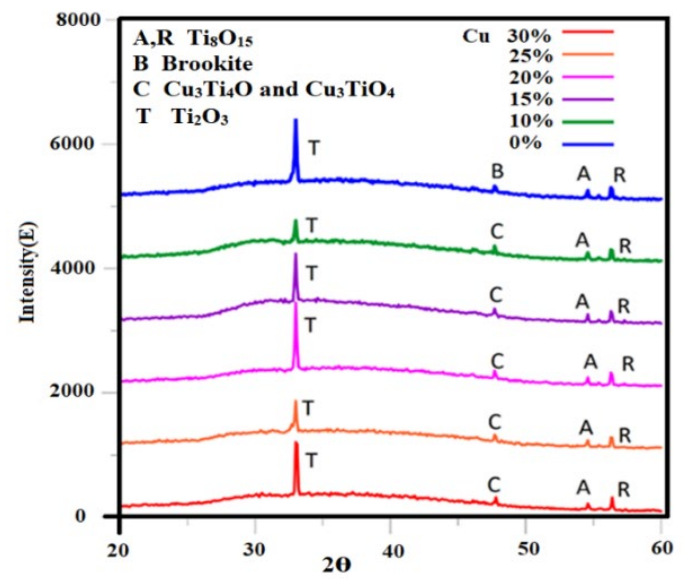

Figure 7. XRD results of pure and doped $\mathrm{TiO}_{2}$ by $\mathrm{Cu}$

\subsection{Optical Characterization}

Fiber optic coupled Ocean HR4000 spectrometer is used to determine the absorption measurements for estimating the band gap of as grown thin films of memristor devices. Tauc plot is plotted by measuring the absorption coefficient $\alpha$ and using the $(\alpha \mathrm{hv})^{n}=B\left(\mathrm{~h} v-E_{g}\right)$ function [22], where, $v$ is the frequency, $h$ is the Planck constant, $B$ is the Boltzmann constant and the band gap is $E_{g}$. The fitted amount of the band gap for several number of measurements of the absorption coefficient for pure and $(10,15,20,25,30 \%) \mathrm{Cu}$ doped $\mathrm{TiO}_{2}$ shown in Fig.7 respectively is $3.389,3.154,2.698,2.653,2.547$ and $2.458 \mathrm{eV}$. The comparison of the fitted amount of the band gap is plotted in this figure. As shown in Tauc plot the band gap is shifted to lower amount of band gap by growing Cu doping level.

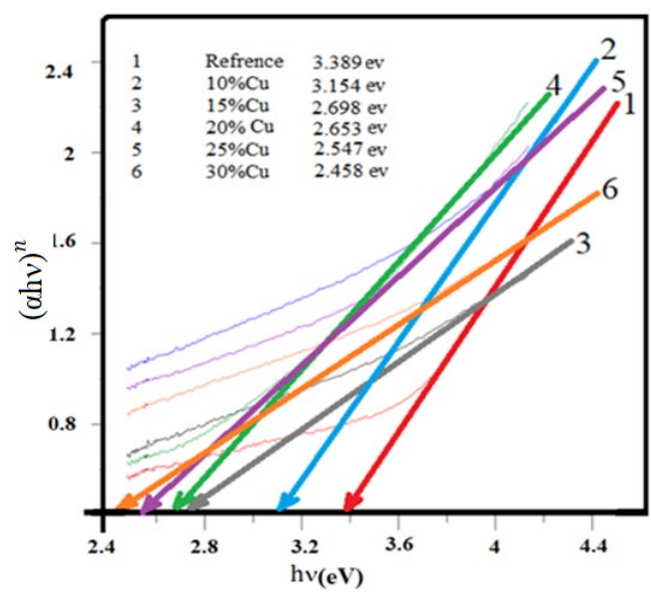




\subsection{AFM Measurement Result}

During $\mathrm{TiO}_{2}$ growing process, $\mathrm{Cu}$ concentration was changed by adjusting the time duration and trigger signal frequency applied to the power supply of $\mathrm{Cu}$ target. Although the measurements related to the exact copper content in the films did not clarified, the effect of copper addition on the surface roughness was investigated by AFM measurements. For comparison all film thickness kept constant at $30 \mathrm{~nm}$. Height distributions for $2 \times 2 \mu \mathrm{m}^{2}$ area scanning is given Fig. 8 , indicate at highest $\mathrm{Cu}$ content peak height is shifted to higher value and distribution range increases.

The surface roughness for the pure $\mathrm{TiO}_{2}$ without any additives is $0.30 \mathrm{~nm}$. Surface roughness is a measure for the $\mathrm{Cu}$ doping effect on $\mathrm{TiO}_{2}$ film surface morphology. The analysis AFM measurements are given in Fig. 9 roughness increased from $0.41 \mathrm{~nm}$ to $0.52 \mathrm{~nm}$.

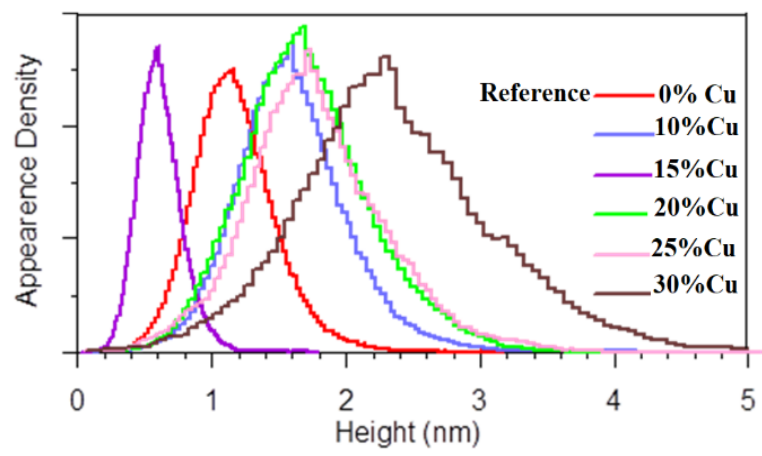

Figure 9. Height distributions of pure and $\mathrm{Cu}$ doped $\mathrm{TiO}_{2}$ thin films as calculated from AFM data.

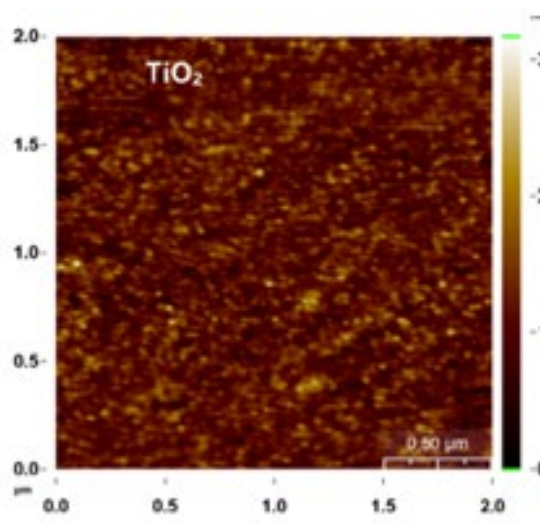

(a)

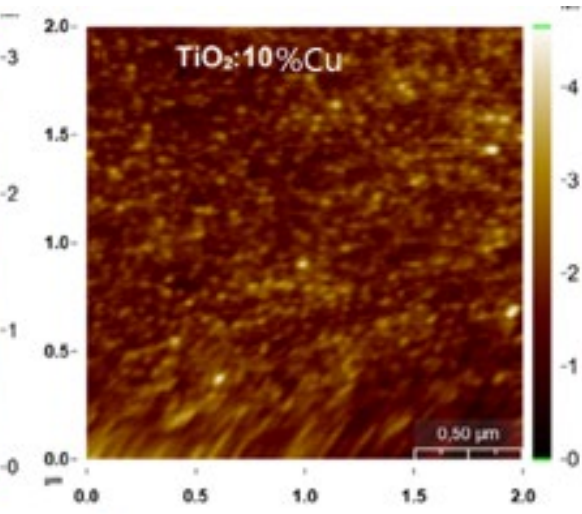

(b)

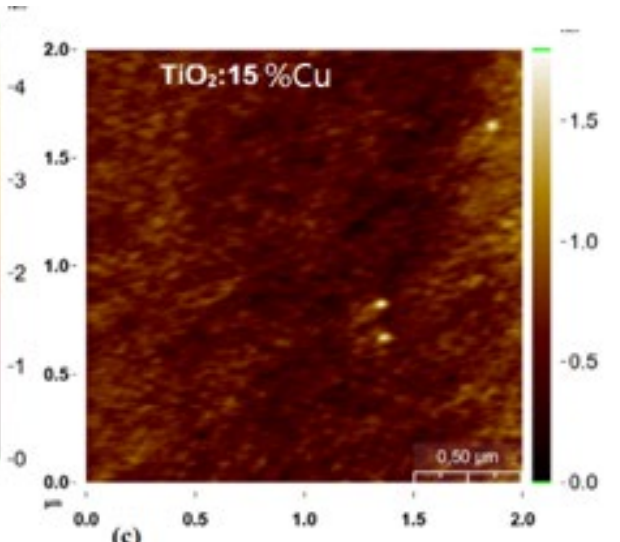

$$
-
$$
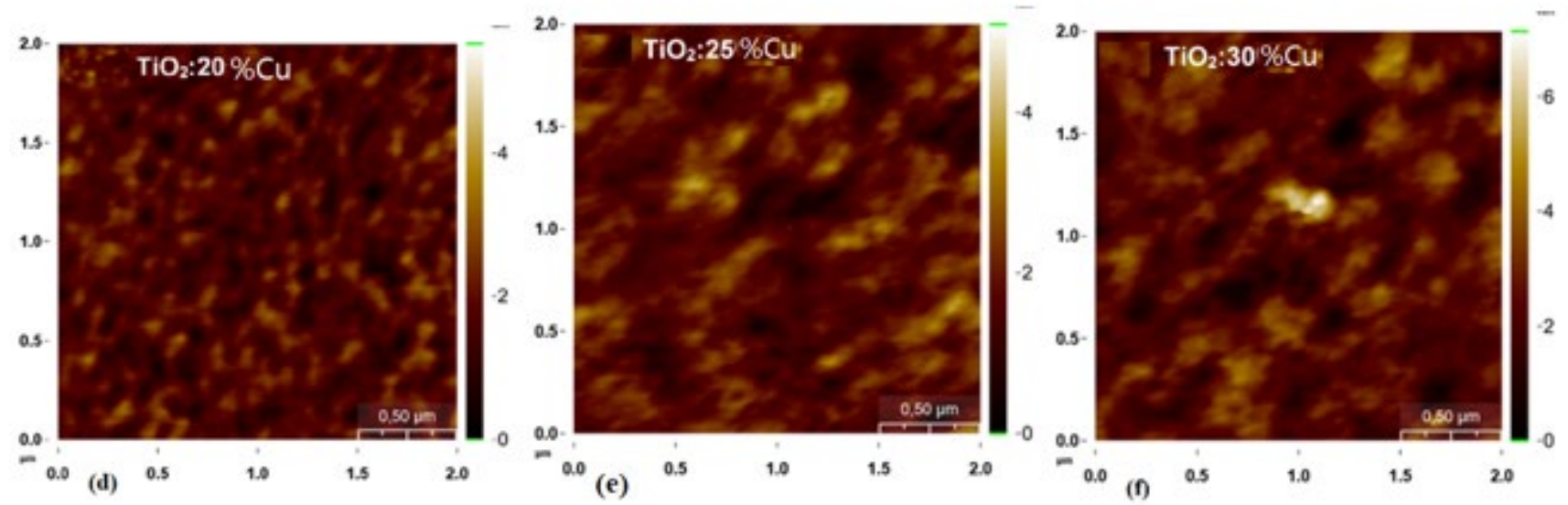

Figure 10. (a, b, c, d, e, f) AFM images showing the surface morphology of 30nm thick $\mathrm{TiO}_{2}$ and Cu doped films on the silicon substrate.

3.Conclusion 


\section{Declaration of Conflict of Interests}

[18.] Sawa, A., Fujii, T., Kawasaki, M. \& Tokura, Y. Interface resistance switching at a few nanometer thick perovskite manganite active layers. Appl. Phys. Lett. (2006), 88, 232-112.

The authors declare that there is no conflict of interest.

[19.] Hamaguchi, M., Aoyama, K., Asanuma, S., Uesu, Y. \& Katsufuji, T Electric-fieldinduced resistance switching universally observed in transition-metal-oxide thin films. Appl. Phys. Lett. (2006), 88, 142-508.

\section{Acknowledgement}

This study was funded by TUBITAK grant number 117F405.

\section{References}

[1.] Chua, L., Memristor-The missing circuit element. IEEE Transactions on Circuit Theory,(1971). 18(5): p. 507-519.

[2.] Meijer, G. I. Science (2008), 319, 1625-1626.

[3.] Waser, R. Aono, M. Nat. Mater. (2007), 6, 833-840.

[4.] Sawa, A. Mater. Today (2008), 11, 28-36.

[5.] Waser, R. Dittmann, R. Staikov, G. Szot, K. Adv. Mater. (2009), 21, 2632-2663.

[6.] Scott, J. C. \& Bozano, L. D. Nonvolatile memory elements based on organic materials. Adv. Mater. (2007),19, 1452-1463.

[7.] Charles P. Collier, Gunter Mattersteig Eric W. Wong,Yi Luo Kristen Beverly, José Sampaio, Françisco M. Raymo, J. Frase Stoddart, James R. Heath ,A [2] catenane-based solid state electronically reconfigurable switch. Science (2000).289, 11721175.

[8.] Zhitenev, N.B., Sidorenko,A., Tennant,D.M., Cirelli,R.A. Chemicalmodification of the electronic conducting states in polymer nanodevices. Nature Nanotechnol. (2007), 2, 237-242.

[9.] Smits, J. H. A., Meskers, S. C. J., Janssen, R. A. J., Marsman, A. W. \& de Leeuw, D. M., Electrically rewritable memory cells from poly(3-hexylthiophene) Schottky diodes. Adv. Mater. (2005), 17 1169-1173.

[10.] Lai, Q. X., Zhu, Z. H., Chen, Y., Patil, S. \& Wudl, F. Organic nonvolatile memory by dopant-configurable polymer. Appl Phys. Lett. 88, (2006), 133-515.

[11.] Terabe, K., Hasegawa, T., Nakayama, T. \& Aono, M.Quantized conductance atomic switch. Nature (2005),433, 47-50.

[12.] Kozicki, M. N., Park, M. \& Mitkova, M. Nanoscale memory elements based on solid-state electrolytes. IEEE Trans. Nanotechnol. (2005), 4, 331-338.

[13.] [Stefan Dietrich, Michael Angerbauer, Milena Ivanov , Dietmar Gogl , Heinz Hoenigschmid , Michael Kund , Corvinet ,et.al. A nonvolatile 2-Mbit CBRAM memory core featuring advanced read and program control. IEEE J. Solid State Circuits (2007), 42 839-845.

[14.] John R. Jameson, Yoshiaki Fukuzumi, Zheng Wang, and Peter Griffin ,Field-programmable rectification in rutile TiO2 crystals. Appl. Phys. Lett. (2007),91, 112 -101.

[15.] Jeong, D. S., Schroeder, H. \& Waser, R., Coexistence of bipolar and unipolar resistive switching behaviors in a $\mathrm{Pt} / \mathrm{TiO} 2 / \mathrm{Pt}$ stack Electrochem. Solid State Lett. (2007),10, G51-G53

[16.] Beck, A., Bednorz, J. G., Gerber, C., Rossel, C. \& Widmer, D Reproducible switching effect in thin. oxide films for memory applications. Appl. Phys. Lett. (2000), 77, 139-141.

[17.] Szot, K., Speier, W., Bihlmayer, G. \& Waser, R. Switching the electrical resistance of individual dislocations in singlecrystalline SrTiO3. Nature Mater. (2006),5, 312-320.

\section{How to Cite This Article}

Arashloo, B. A., Efeoğlu, H.,, Cupper doping effect on the electrical characteristics of $\mathrm{TiO}_{2}$ based Memristor, Brilliant Engineering, 1(2021), 19-24.
[20.] Strukov, D. B. \& Likharev, K. K. Defect-tolerant architectures for nanoelectronic crossbar memories. J. Nanosci. Nanotechnol. (2007), 7, 151-167.

[21.] Dmitri B. Strukov, Gregory S. Snider, Duncan R. Stewart, R. Stanley Williams, The missing memristor found.Nature,(2008),453:p.80.

[22.] Nan Shi , Xiaohui Li , Tongxiang Fan, Han Zhou, Jian Ding , Di Zhang and Hanxing Zhu., Biogenic N-I-codoped TiO2 photocatalyst derived from kelp for efficient dye degradation. Energy \& Environmental Science, (2011), 4(1): p. 172-180.

[23.] Jeong, D. S. Kim, K. M. Kim, S. Choi, B. J. Hwang, C. S. Memristors for Energy-Efficient New Computing Paradigms. Adv. Electron. Mater. (2016), 2, 1600090.

[24.] Li, Y Wang, Z. Midya, R. Xia, Q. Yang, J. J. Review of Memristor Devices in Neuromorphic Computing: Materials Sciences and Device Challenges. J. Phys. D: Appl. Phys. (2018), 51, 503002.

[25.] Jeong, Y. Lu, W. Neuromorphic Computing Using Memristor Crossbar Networks: A Focus on Bio-Inspired Approaches. IEEE Nanotechnoly. Mag. (2018), 12,6-18.

[26.] Burr, G. W. Shelby, R. M. Sebastian, A. Kim, S. Kim, S. Sidler, S. Virwani, K. Ishii, M. Narayanan, P. Fumarola, A. Sanches, L. L. Boybat, I. Le Gallo, M. Moon, K. Woo, J. Hwang, H. Leblebici, Y. Neuromorphic computing using non-volatile memory. Adv. Phys. (2017), 2, 89-124.

[27.] Xiaobing Yan, Jianhui Zhao, Sen Liu, Zhenyu Zhou, Qi Liu, Jingsheng Chen, and Xiang Yang Liu. Memristor with AgCluster-Doped TiO2 Films as Artificial Synapse for Neuroinspired Computing. Adv. Funct. Mater. (2017), 1705320,1-9.

[28.] Yaoyuan Wang, Ziyang Zhang, Mingkun Xu, Yifei Yang, Mingyuan $\mathrm{Ma}$, Huanglong Li, Jing Pei, and Luping Shi. SelfDoping Memristors with Equivalently Synaptic Ion Dynamics for Neuromorphic Computing. ACS Appl. Mater. Interfaces. (2019), 11, 24230-24240.

[29.] Z. Wang, S. Joshi, S. E. Savel'ev, H. Jiang, R. Midya, P. Lin, M. Hu, N. Ge, J. P. Strachan, Z. Li, Q. Wu, M. Barnell, G. Li, H. L. Xin, R. S. Williams, Q. Xia, J. J. Yang, Memristors with diffusive dynamics as synaptic emulators for neuromorphic computing.Nat. Mater. (2017), 16, 101

[30.] Yang, J. J. Strukov, D. B. Stewart, D. R. Memristive Devices for Computing. Nat. Nanotechnol. (2013), 8, 13-24.

[31.] Orji, N. G. Badaroglu, M. Barnes, B. M. Beitia, C. Bunday, B. D. Celano, U. Kline, R. J. Neisser, M. Obeng, Y. Vladar, A. E. Metrology for the Next Generation of Semiconductor Devices. Nat. Electron. (2018), 1, 532-547.

[32.] Minkyu You, Tae Geun Kim,and Yun-Mo Sung, Synthesis of CuDoped TiO2 Nanorods with Various Aspect Ratios and Dopant Concentrations,(2010),983-987. 